\title{
MANEJO DE FÍSTULAS NASOALVEOLARES CON LA COLOCACIÓN DE ALOINJERTO, XENOINJERTO YMEMBRANA PARA REGENERACIÓN TISULAR. REVISIÓN DE LA LITERATURA Y PRESENTACIÓN DE TRES CASOS CLÍNICOS
}

Management of nasoalveolar fistulas with placement of allograft, xenograft and membrane for tissue regeneration. Review of the literature and presentation of three clinical cases

Fecha de Recepción: 2 de junio 2021
Tratamento das fístulas nasoalveolares com colocação de aloenxerto, xenoenxerto e membrana para regeneração do tecido. Revisão da literatura e apresentação de três casos clínicos

Aceptado para su publicación: 16 de agosto 2021

\author{
Autores: \\ Mauricio González Rebattú y González 1,a, \\ Ana María Nieto Munguía ${ }^{2, a, f}$ \\ Emmanuel Hernández Pérez ${ }^{1, c, g}$ \\ Christian Adolfo San Juan González 1,b \\ Mauricio García Nucamendi $1, d, g$ \\ Isai Daniel García Montes 1,c,g \\ Jesús Lorenzo Derat Araujo 1,e,g
}

1. Servicio de Cirugía Oral y Maxilofacial. Hospital Regional " $1{ }^{\circ}$ de Octubre". Instituto de Seguridad y Servicios Sociales de los Trabajadores del Estado (ISSSTE). Ciudad de México,

México.

2. Servicio de Cirugía Oral y Maxilofacial. Hospital Regional "Licenciado Adolfo López Mateos". Instituto de Seguridad y Servicios Sociales de los Trabajadores del Estado (ISSSTE). Ciudad de México, México. a. Especialista en Cirugía Oral y Maxilofacial (Universidad

Nacional Autónoma de México).

b. Cirujano Oral y Maxilofacial (egresado del Hospital Regional $1^{\circ}$ de Octubre, ISSSTE).

c. Cirujano dentista (Universidad Nacional Autónoma de México).

d. Cirujano Dentista (Universidad de Ciencias y Artes de

Chiapas, México).

e. Cirujano dentista (Centro de Estudios Universitarios Superiores. Culiacán, Sinaloa México).

f. Médico Adscrito.

g. Residente de Cirugía Oral y Maxilofacial.

Correspondencia: González Rebattú y González, Mauricio. Hospital Regional $1^{\circ}$ de Octubre, ISSSTE. Servicio de Cirugía Maxilofacial. Av. Instituto Politécnico Nacional No 1669. Col. Magdalena de las Salinas, C.P. 07300 Alcaldía Gustavo A. Madero, México, D.F.

$+525555866011$

Correo electrónico: mauriciorebattu@yahoo.com.mx

Conflicto de intereses: los autores declaran no tener conflictos de interés.

\section{Resumen}

Las fístulas nasoalveolares representan una de las secuelas más frecuentes del tratamiento de labio y paladar hendido. Es necesario su tratamiento para favorecer la fonación, la erupción de los órganos dentarios al crear una guía ósea, permitir la separación de la cavidad oral de la nasal y permitir la colocación de implantes dentales que favorezca la oclusión. Los tratamientos que se han establecido son diferentes, pero el injerto autólogo de cresta ilíaca es la primera opción en la mayoría de los casos. El objetivo de este trabajo fue presentar el manejo de fístulas nasoalveolares en el servicio de Cirugía Maxilofacial del Hospital Regional $1^{\circ}$ Octubre, con la presentación de tres casos clínicos, dos pacientes masculinos de 8 y 53 años y un paciente femenino de 16 años, con fístulas nasoalveolares como secuelas de labio y paladar hendido, así como ausencias de incisivos laterales superiores en el sitio del defecto. Para el tratamiento, se ejecutó protocolo quirúrgico para realización de aloinjerto de bloque óseo tricortical, xenoinjerto de matriz ósea desmineralizada y colocación de matriz dérmica acelular. Esta alternativa a la toma de injertos autólogos, disminuyó las comorbilidades de la toma de los autoinjertos y elevó la calidad de vida de los pacientes al realizarse el cierre de las fístulas nasoalveolares.

Palabras clave: fístula oral; cavidad nasal; aloinjertos; xenoinjertos; osteogénesis (fuente: DeCS BIREME).-

\section{Abstract \\ Nasoalveolar fistulas represent one of the most frequent sequelae of cleft lip and palate treatment. Their treat- ment is necessary to aid phonation and the eruption of dental organs by creating a bony guide, to allow the separation of the oral cavity from the nasal cavity and}




\section{REFO}

VOL. XIV | N² 2| AÑO 2021

ISSN $1668-7280$

ISSN-E 2683-7986 to allow the placement of dental implants that help occlusion. Different treatments have been administered. The first choice in most cases is autologous iliac crest grafting. This study aimed to present the treatment of nasoalveolar fistulas in the Maxillofacial Surgery Service of the Hospital Regional $1^{\circ}$ Octubre. Three clinical cases are presented: two male patients aged 8 and 53 years and one female patient aged 16 years, with nasoalveolar fistulas as sequelae of cleft lip and palate, as well as the absence of upper lateral incisors at the site of the defect. The treatment consisted of using a surgical protocol to perform tricortical bone block allograft, demineralized bone matrix xenograft and acellular dermal matrix placement. This alternative to taking autologous grafts reduced their comorbidities and improved the patients' quality of life by closing the nasoalveolar fistulas.

Key words: oral fistula; nasal cavity; allografts; heterografts; osteogenesis (source: MeSH NLM)

\section{Resumo}

As fístulas nasoalveolares representam uma das sequelas mais frequentes do tratamento da fissura labiopalatina. O seu tratamento é necessário para promover a fonação, a erupção dos órgãos dentais a través da criação de uma guia óssea, permitindo a separação da cavidade oral da nasal e permitindo a colocação de implantes dentários que promovem a oclusão. Os tratamentos instituídos são diferentes, mas o enxerto autólogo de crista ilíaca é a primeira opção na maioria dos casos. O objetivo deste trabalho foi apresentar o manejo das fístulas nasoalveolares no serviço de Cirurgia Maxilofacial do Hospital Regional $1^{\circ}$ Octubre, com a apresentação de três casos clínicos, sendo dois pacientes do sexo masculino com idades entre 8 e 53 anos e uma paciente do sexo feminino com 16 anos, com fístulas nasoalveolares como sequela de fissura labiopalatina, além da ausência de incisivos laterais superiores no local do defeito. Para o tratamento, foi realizado protocolo cirúrgico para realização de aloenxerto de bloco ósseo tricortical, xenoenxerto de matriz óssea desmineralizada e colocação de matriz dérmica acelular. Essa alternativa à realização de enxertos autólogos diminuiu as comorbidades da realização de autoenxertos e aumentou a qualidade de vida dos pacientes após o fechamento das fístulas nasoalveolares.

Palavras-chave: fístula bucal;cavidade nasal; loenxertos; xenoenxertos; osteogênese (fonte: DeCS BI REME)

\section{Introducción}

La formación de la cara se da entre la cuarta y octava semana de vida intrauterina. Se desarrolla a partir de cinco primordios faciales, los cuales surgen alrededor del estomodeo o cavidad primitiva hacia la quinta semana de vida intrauterina. Iniciando con la formación del paladar primero y por consecuente el labio superior, la columnela nasal, el alvéolo maxilar y la premaxila ${ }^{1}$.

La unión del paladar primario y el paladar secundario se inicia en la novena semana de vida intrauterina, con el evento de descenso de la lengua, y para la décima semana ya se encuentra la fusión completa ${ }^{2}$.

Los defectos de la fusión en línea media de los procesos nasales mediales originarán fisuras labiales, e igualmente, los defectos en la fusión de los procesos palatinos darán origen a fisuras palatinas. Es más frecuente encontrar estos dos defectos juntos, sin embargo, también pueden encontrarse por separado, y pueden estar o no asociados a síndromes ${ }^{3}$.

Las fisuras no asociadas a síndromes pueden estar relacionadas a la combinación de factores ambientales con ciertos genes, se ha encontrado también asociación con el consumo de bebidas alcohólicas, consumo de tabaco y los medicamentos anticonvulsivantes durante el periodo de gestación aumenta la incidencia ${ }^{3}$.

El manejo quirúrgico de pacientes con fisura labial y palatina consta de varias fases según la edad y etapa de desarrollo en la que se encuentre el paciente y requiere de un tratamiento integral multidisciplinario. El esquema recomendado inicia a los 3 meses con la queiloplastía primaria para las fisuras labiales unilaterales o bilaterales, seguido de la palatoplastía unilateral o bilateral alrededor de los 8 y 12 meses de vida. La próxima cirugía se recomienda realizarla entre los 6 y 12 años, para corregir secuelas. finalmente en la adolescencia se realiza cirugía ortognática y rinoplastia secundaria. Una de las secuelas encontradas frecuentemente son las fístulas nasoalveolares, comunicación entre la cavidad nasal y la cavidad oral que debe cerrarse de manera quirúrgica ${ }^{2}$. 
Revista de la Facultad de Odontología González Rebattú y González | Nieto Munguía | Hernández Pérez San Juan González | García Nucamendi | García Montes | Derat Araujo

\section{REFO}

VOL. XIV | N² 2| AÑO 2021

ISSN 1668-7280

ISSN-E 2683-7986
Boyne y Sands en 1961, establecieron las etapas para el cierre de fístulas con colocación de injerto: 1) injerto óseo primario: pacientes menores de 2 años, 2) injerto óseo secundario temprano: pacientes entre los 2 y los 5 años, injerto óseo secundario: pacientes entre 5 y 16 años, injerto óseo secundario tardío: pacientes mayores de 16 años 4 .

Los injertos se clasifican en: 1) aloinjerto: compuestos de tejidos tomados de un individuo de la misma especie, pero genéticamente diferentes; 2) xenoinjerto: compuestos de tejidos tomados de un donador de otra especie; 3) autoinjerto: compuestos por tejidos tomados del mismo individuo; 4) aloplásticos: compuestos de materiales sintéticos y/o naturales como el fosfato tricálcico ${ }^{5}$.

El objetivo de este trabajo fue presentar tres casos clínicos con fístulas nasoalveolares tratadas con aloinjerto de bloque óseo tricortical, xenoinjerto de matriz ósea desmineralizada y colocación de matriz dérmica acelular.

\section{Casos clínicos}

A continuación, se describe la técnica quirúrgica, siendo la misma para los tres pacientes, con la diferencia que, en el paciente masculino de 53 años se realizó la fijación del bloque de hueso tricortical con un tornillo de osteosíntesis para lograr mayor estabilidad debido a la extensión del defecto. Los pacientes fueron intervenidos quirúrgicamente en el período del mes de diciembre del año 2018 al mes de febrero del año 2020 en el Hospital Regional $1^{\circ}$ de Octubre, ISSSTE (México).

Previo a técnicas de asepsia y antisepsia se procede a la infiltración de lidocaína al $2 \%$ con epinefrina 1:100 000 en región de mucosa palatina, encía y mucosa vestibular perilesional de fístula nasoalveolar. Posteriormente, se realizaron las incisiones para el levantamiento del colgajo tipo Newman, se disecó la mucosa nasal y se realizó el cierre de la fístula de la mucosa nasal con sutura vicryl 4-0 (Figuras 1 y 2 ).

Se procedió a la realización del diseño del aloinjerto tricortical con ayuda de fresa $703 \mathrm{~L}$ y fresón de pera para lograr la adecuada posición de este, posteriormente se realizó el relleno de los espacios del defecto con $5 \mathrm{cc}$ de xenoinjerto de matriz ósea desmineralizada y la posterior confección y colocación de una matriz dérmica acelular para cubrir los injertos óseos realizados, se verificó la hemostasia y se efectuó la sutura con puntos simples de nylon 4-0 (Figuras 3-6).

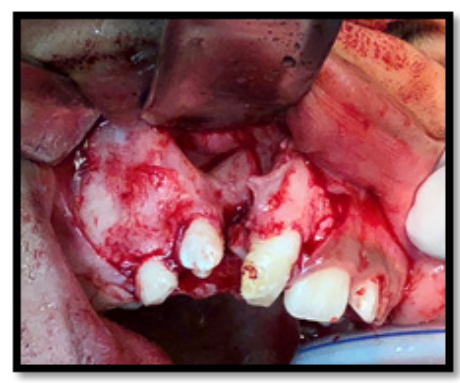

Figura 1. Levantamiento de colgajo tipo Newman, se observa defecto óseo de fístula nasoalveolar derecha.

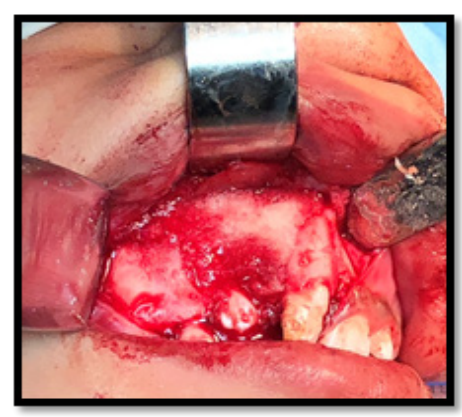

Figura 4. Relleno del defecto óseo con xenoinjerto de matriz ósea desmineralizada.

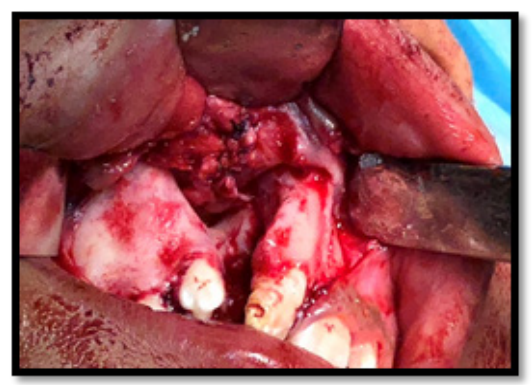

Figura 2. Disección de mucosa nasal, realización de cierre fístula nasal con Vicryl 4-0.

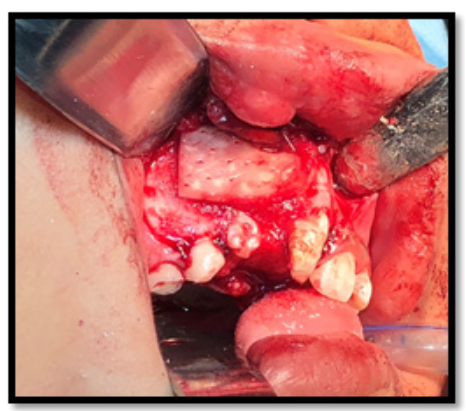

Figura 5. Colocación de matriz dérmica acelular para cubrir los injertos óseos.

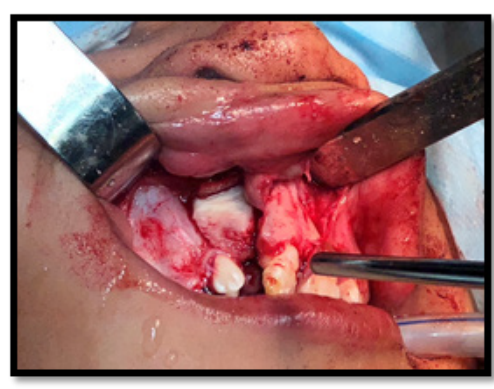

Figura 3. Colocación del injerto tricortical en el defecto óseo.

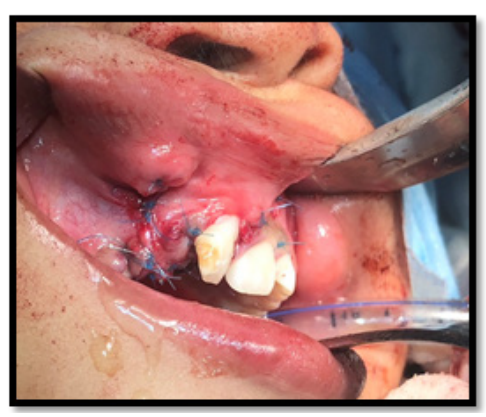

Figura 6. Colocación de matriz dérmica acelular para cubrir los injertos óseos. 


\section{Caso clínico 1}

Paciente masculino de 8 años de edad, originario y residente de la Ciudad de México, sin antecedentes heredofamiliares de importancia para el padecimiento actual, con antecedentes personales patológicos de labio y proceso alveolar hendido unilateral derecho, cierre primario de labio a los 3 meses de edad y cierre de dehiscencia de labio a los 2 años de edad. Presentó como secuela fístula nasoalveolar derecha (Figuras 7 y 8). Se realizó protocolo quirúrgico para realización de aloinjerto de bloque óseo tricortical, xenoinjerto de matriz ósea desmineralizada y colocación de matriz dérmica acelular. Se realizó tomografía computada (TC) de macizo facial postoperatoria inmediata mostrando la adecuada posición del aloinjerto (Figura 9), así como ortopantomografía de control a una semana de la intervención quirúrgica. Se realizaron fotografías clínicas a 11 meses del procedimiento quirúrgico mostrando la ausencia de la fístula nasoalveolar, así como ortopantomografía de control a 11 meses de la intervención quirúrgica donde se evidenció la formación ósea en el defecto y la adecuada erupción del órgano dentario 13 (Figura 10).

\section{Caso clínico 2}

Paciente femenino de 16 años de edad, originaria y residente de la Ciudad de México, con antecedentes heredofamiliares positivos (prima materna) de labio y paladar hendido unilateral izquierdo, con antecedentes personales patológicos de labio y proceso alveolar hendido del lado izquierdo, cierre primario de labio a los 3 meses de edad, cirugía a los 14 años por queiloplastía, presentándose como secuela fístula nasoalveolar (Figuras 11 y 12). Se realizó protocolo quirúrgico para aloinjerto de bloque óseo tricortical, xenoinjerto de matriz ósea desmineralizada y colocación de matriz dérmica acelular. Se efectuó

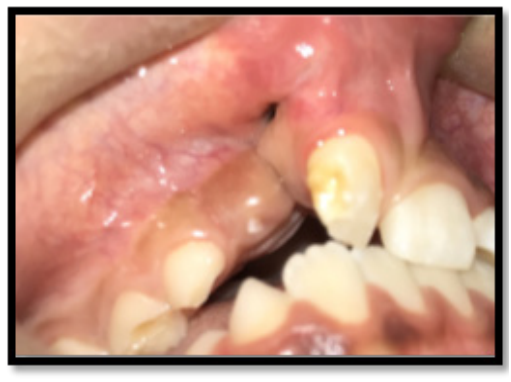

Figura 7. Fotografía intraoral de fístula nasoalveolar derecha.

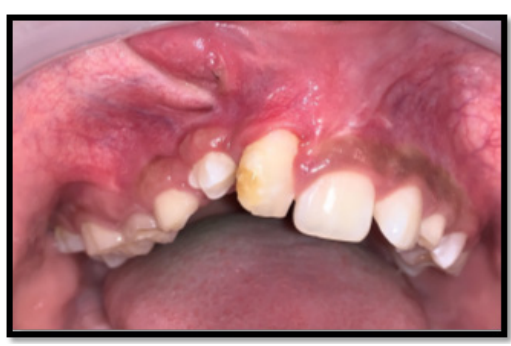

Figura 10. Fotografía clínica intraoral a 11 meses del procedimiento quirúrgico, ausencia de la fístula nasoalveolar derecha.

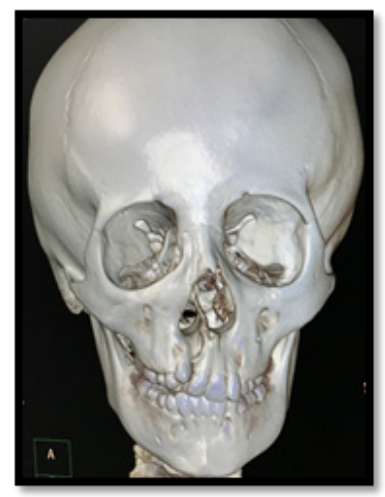

Figura 8. Reconstrucción 3D de TC de macizo facial, donde se observa fístula nasoalveolar derecha.

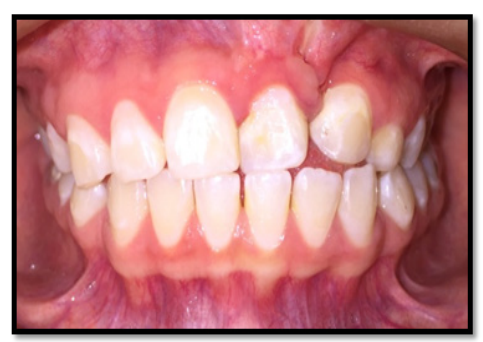

Figura 11. Fotografía intraoral de fístula nasoalveolar izquierda.

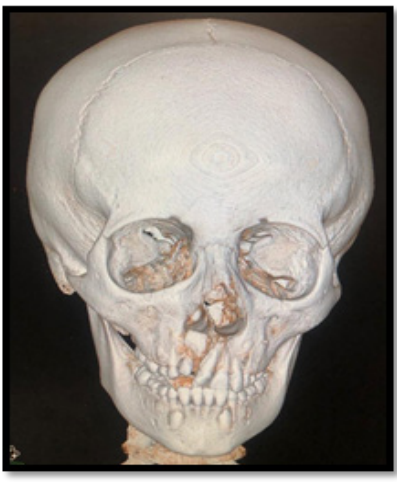

Figura 9. TC de macizo facial postoperatoria inmediata.

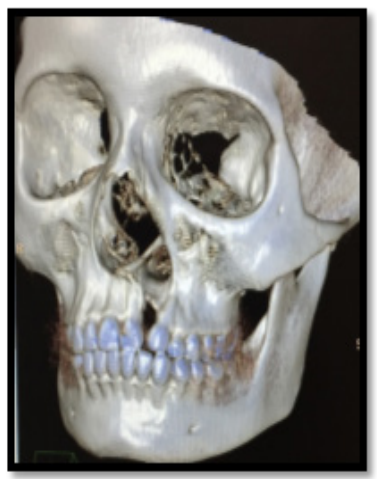

Figura 12. Reconstrucción 3D de TC de macizo facial, donde se observa fístula nasoalveolar izquierda. 


\section{REFO}

VOL. XIV | N 2| AÑO 2021

ISSN 1668-7280

ISSN-E 2683-7986 ortopantomografía postoperatoria a los 3 meses del procedimiento quirúrgico mostrando la adecuada posición del aloinjerto (Figura 13). Se realizó fotografía clínica a 10 meses del procedimiento quirúrgico que mostró la ausencia de la fístula nasoalveolar, donde se observó la adecuada colocación de aparatología de ortodoncia para la corrección de la oclusión dental (Figura 14).

\section{Caso clínico 3}

Paciente masculino de 53 años de edad, originario y residente de la Ciudad de México, sin antecedentes heredofamiliares de importancia para el padecimiento actual, con antecedentes personales patológicos de labio y proceso alveolar hendido unilateral izquierdo, cierre primario de labio a los 15 años de edad. Presentó como secuela fistula nasoalveolar (Figuras 15 y 16). Se realizó protocolo quirúr- gico para la realización de aloinjerto de bloque óseo tricortical fijado con tornillo de osteosíntesis (Figura 17), xenoinjerto de matriz ósea desmineralizada y colocación de matriz dérmica acelular. Acude a cita de control a los 3 meses con seguimiento radiográfico, donde se observa adecuada integración de injerto en bloque tricortical y tornillos de osteosíntesis en adecuada posición y función.

\section{Discusión}

El manejo quirúrgico para el cierre de las fístulas nasoalveolares es muy controvertido, dado que se han propuesto diversas técnicas, materiales e injertos. El estándar de oro es el injerto autólogo de cresta iliaca, pero puede presentar diversas complicaciones como las fracturas, hemorragias, parestesia, infecciones, dehiscencias de las heridas y recuperación tardía ${ }^{6}$.

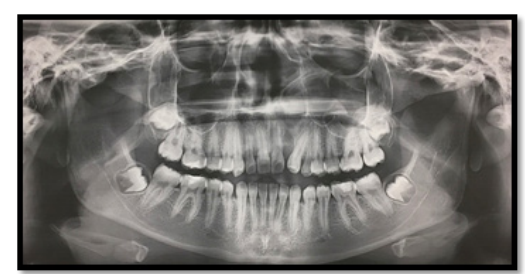

Figura 13. Ortopantomografía de control a 3 meses de la intervención quirúrgica, evidencia radiopaca de formación ósea en el sitio del defecto.

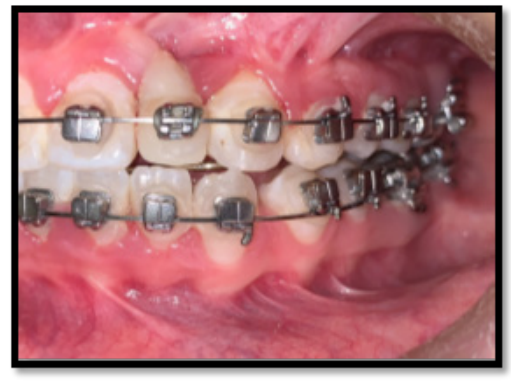

Figura 14. Fotografía clínica intraoral a 10 meses del procedimiento quirúrgico, ausencia de la fístula nasoalveolar izquierda y aparatología de ortodoncia para corrección de colapso transversal maxilar.

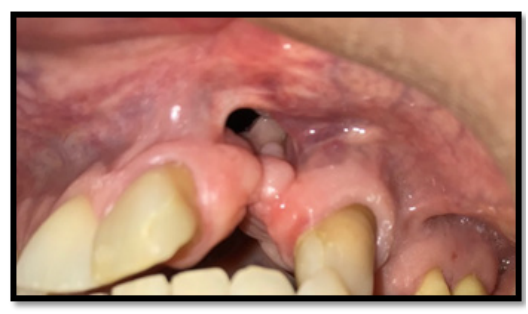

Figura 15. Fotografía intraoral de fístula nasoalveolar izquierda.

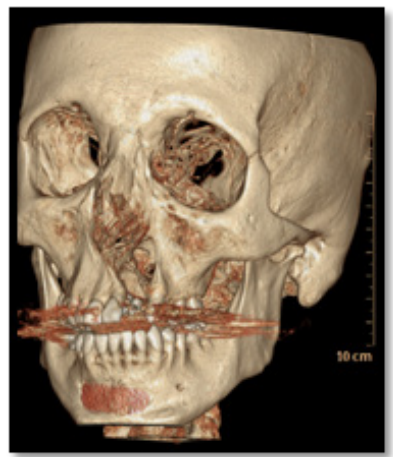

Figura 16. Reconstrucción 3D de TC de macizo facial, donde se observa fístula nasoalveolar izquierda.

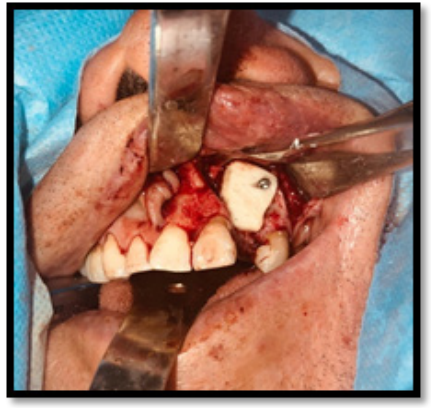

Figura 17. Colocación del injerto tricortical en el defecto óseo fijado con tornillo de osteosíntesis. 
Manejo de Fístulas nasoalveolares con la colocación de aloinjerto, xenoinjerto ymembrana para regeneración tisular. Revisión de la literatura y presentación de tres casos clínicos de aloinjerto, xenoinjerto ymembrana para regeneración tisular. Revisión de la literatura y presentación de tres casos clínicos

\section{REFO}

VOL. XIV | N² 2| AÑO 2021

ISSN 1668-7280

ISSN-E 2683-7986
Los pacientes con secuelas de labio y paladar hendido, deben ser intervenidos en las edades más adecuadas conforme a los protocolos. Según Boyne y Sands ${ }^{7}$, un injerto óseo secundario es adecuado ya que se realiza en la dentición mixta, con edades entre los ocho y los 12 años, previo a la erupción del canino. Idealmente el injerto debe realizarse cuando el desarrollo radicular del canino se encuentra en la mitad o tres cuartos del total ${ }^{8}$. Se considera el protocolo adecuado, ya que el crecimiento sagital y transverso del maxilar se completa aproximadamente a los ocho años de edad y el crecimiento vertical faltante se origina con la dentición definitiva y la erupción del canino?.

El injerto secundario es predecible en el tiempo y seguro en sus resultados, por esta razón es el más utilizado, su mayor ventaja es permitir la erupción del canino a través del injerto, mejorando su estabilidad en la arcada dentaria. Este tipo de injerto ha demostrado tener menor repercusión en el crecimiento, ya que previene el colapso transversal del maxilar. Sin embargo, dentro de sus desventajas se encuentra la reabsorción ósea ${ }^{10}$. Además, un punto clave para el éxito de un injerto óseo secundario es contar con un especialista en ortodoncia, para aproximar los segmentos maxilares con aparatología antes de la cirugía. Un adecuado protocolo nos permite el cierre de las fístulas nasoalveolares, la correcta erupción dental por medio de una guía ósea cuando se repara el defecto, la posibilidad de una posterior cirugía para la colocación de implantes dentales y la separación de la cavidad oral y nasal con el cierre de la fístula ${ }^{8}$.

El avance de los biomateriales nos ha permitido que el uso de la matriz ósea desmineralizada proveniente del xenoinjerto y un aloinjerto como el bloque óseo tricortical, nos asegure la formación de hueso a través de los procesos de la osteoinducción y la osteoconducción, sin incrementar la tasa de comorbilidades cuando se realiza la toma de un injerto autólogo 9,10. El protocolo que utilizamos en los casos clínicos para el manejo de las fistulas nasoalveolares, ha logrado una considerable tasa de éxito a lar- go plazo. La alternativa de tratamiento propuesta, evitó las comorbilidades que conlleva la toma de injertos autólogos y mejoró la calidad de vida de nuestros pacientes, ya que se logró el objetivo del cierre de las fístulas nasoalveolares así como la adecuada conformación del reborde maxilar.

\section{Referencias Bibliográficas}

1. Navarro Gasparetto CE, Bardales Lasteros A. Atlas de Fisura Labiopalatina Primaria. Lima, Perú: MAD CORP; 2000.

2. Rozen-Fuller I. Labio y paladar hendido. Conceptos básicos. 2da. ed. México: Casa de Vacunas; 2000.

3. Neville BW, Damm DD, Allen CM, Chi AC. Oral and Maxillofacial Pathology. 4th ed. St. Louis, Missouri: Elsevier; 2016.

4. Turvey TA, Vig KWL, Fonseca RJ. Facial Clefts and Craniosynostosis. Principles and Management. Philadelphia: WB Saunders; 1996.

5. Boyne PJ. Bone Grafting in the Osseous Reconstruction of Alveolar and Palatal Clefts. Oral Maxillofac Surg Clin North Am. 1991;3(3):589-597. doi:10.1016/S10423699(20)30529-X

6. Kalk WW, Raqhoebar G, Jansma J, Boering G. Morbidity from iliac crest bone harvesting. J Oral Maxillofac Surg. 1996;54(12):1424-1429. doi: 10.1016/S02782391(96)90257-8

7. Boyne PJ, Sands NR. Secondary bone grafting of residual alveolar and palatal clefts. J Oral Surg.1972;30(2):87-92. 8. Uribe Fenner F, Castellón Zirpel L, Laissle CG. Erupción del canino permanente a través de injertos óseos alveolares secundarios en pacientes con fisuras labiopalatinas. Rev Odontol Mex. 2007;11(4):207-214. Disponible en: https://www.medigraphic.com/pdfs/odon/uo-2007/ uo074g.pdf

9. Víctor BA, Pérez GA, Telich TJE, Chávez PG, de la Concha BE, Garza LH. Injerto óseo alveolar y su importancia en los pacientes con labio y paladar hendido. Cir Plast. 2017; 27(1):31-37. Disponible en: https://www.medigraphic.com/pdfs/cplast/cp-2017/cp171g.pdf

10. Hernández Nava DL, López Noriega JC, Bernal Faro RF. Cierre de fístula nasoalveolar con aspirado medular e injerto alogénico. Presentación de caso clínico. Rev Mex Cir Bucal Maxilofac. 2012;8(2):40-44. Disponible en: https://www.medigraphic.com/pdfs/cirugiabucal/cb-2012/ cb122b.pdf 